\title{
Weak-localization effect in superconductors from radiation damage
}

\author{
Mi-Ae Park \\ Department of Physics, University of Puerto Rico at Humacao, Humacao, Puerto Rico 00791 \\ Yong-Jihn Kim \\ Department of Physics, Bilkent University, 06533 Bilkent, Ankara, Turkey \\ (Received 16 September 1999; revised manuscript received 3 November 1999)
}

\begin{abstract}
Large reductions of the superconducting transition temperature $T_{c}$ and the accompanying loss of the thermal electrical resistivity (electron-phonon interaction) due to radiation damage have been observed for several $A 15$ compounds, Chevrel phase and ternary superconductors, and $\mathrm{NbSe}_{2}$ in the high-fluence regime. We examine these behaviors based on a recent theory of the weak localization effect in superconductors. We find a good fitting to the experimental data. In particular, the weak localization correction to the phonon-mediated interaction is derived from the density correlation function. It is shown that weak localization has a strong influence on both the phonon-mediated interaction and the electron-phonon interaction, which leads to the universal correlation of $T_{c}$ and the resistance ratio.
\end{abstract}

\section{INTRODUCTION}

Much attention has been paid to experimental and theoretical investigations of the radiation effects on superconductors. ${ }^{1-3}$ For practical applications of the superconductors in the magnet coils of a fusion reactor, the radiation response of the materials is important because they are subjected to irradiation. On the theoretical side, the disorder effects in superconductors caused by irradiation are interesting. The radiation effects in elemental type II superconductors showed that the superconducting transition temperature $T_{c}$ does not change significantly for relatively high-fluence irradiations. ${ }^{3}$ The slight reduction was attributed to the reduction of the gap anisotropy. Subsequent annealing leads to a partial recovery of the $T_{c}$ changes, implying the importance of the microscopic details of the disorder structures. We note that most elemental type II superconductors are radiation tolerant. For instance, the $\mathrm{He}-4$ dose which resulted in $\Delta \rho_{0} \sim 100 \mu \Omega \mathrm{cm}$ in $\mathrm{Nb}_{3}$ Ge causes $\Delta \rho_{0} \sim 2 \mu \Omega \mathrm{cm}$ in Nb. ${ }^{4}$ Here $\rho_{0}$ denotes the residual resistivity. On the other hand, $A 15$ compounds show universal large reductions of $T_{c}$ and critical currents $I_{c}$ for high-fluence irradiations. ${ }^{4-14}$ The residual resistivity $\rho_{0}$ also increases over $\sim 100 \mu \Omega \mathrm{cm}$, indicating that $A 15$ compounds are radiation susceptible. Note that the layered compound $\mathrm{NbSe}_{2}$, ternary superconductors $\mathrm{LuRh}_{4} \mathrm{~B}_{4}$ and $\mathrm{ErRh}_{4} \mathrm{~B}_{4},{ }^{15}$ and Chevrel phase superconductors, ${ }^{3}$ such as $\mathrm{PbMo}_{6} \mathrm{~S}_{8}, \mathrm{PbMo}_{6} \mathrm{~S}_{7}$, and $\mathrm{SnMo}_{5} \mathrm{~S}_{8}$, also show the large $T_{c}$ reduction in the highfluence regime.

In this paper we propose an explanation of the microscopic mechanism underlying the universal large reductions of $T_{c}$ and $I_{c}$ in $A 15$ compounds and other materials. In particular, we stress the experimental observation of the correlation between the electrical-resistance ratio and $T_{c} \cdot{ }^{4,8,9,11}$ Testardi and co-workers ${ }^{4,8,9}$ considered $\mathrm{Nb}-\mathrm{Ge}, \mathrm{V}_{3} \mathrm{Si}$, and $\mathrm{V}_{3} \mathrm{Ge}$ for a variety of samples produced with differing chemical composition, preparation conditions, and with varying amounts of ${ }^{4} \mathrm{He}$-induced defects. They found a close relation between the resistance ratio and $T_{c}$ for those samples irrespective of the manner how the disorder was achieved. Furthermore, they found that decreasing $T_{c}$ is accompanied by a decrease of the thermal electrical resistivity (electronphonon interaction). ${ }^{4}$ It was also reported that tunneling experiments in $\mathrm{Nb}_{3} \mathrm{Ge}$ and $\mathrm{Nb}-\mathrm{Sn}$ clearly show a decrease of the electron-phonon coupling constant $\lambda$ accompanying the decrease of $T_{c}$ with disorder. ${ }^{16-18}$

However, previous theoretical studies focused not on changes of the electron-phonon interaction but on the smearing of the electronic density of states near the Fermi level $N\left(E_{F}\right)$, the microscopic details of the disorder, the gap anisotropy, and the enhancement of the Coulomb repulsion. ${ }^{19-25}$ It is obvious that a consistent explanation of the existing experimental data was not possible in those prior theories. Recently, Kim and Overhauser ${ }^{26}$ pointed out that Anderson's theorem ${ }^{27}$ is valid only to the first power in the impurity concentration and the phonon-mediated interaction decreases exponentially by Anderson localization, in agreement with the above experimental findings. As expected, it was shown that the same weak localization correction terms occur in both the conductivity and phonon-mediated interaction. ${ }^{28,29}$ Based on the reduced phonon-mediated interaction, we propose an explanation of the universal reductions of $T_{c}$ and $I_{c}$ and the universal correlation of $T_{c}$ and resistance ratio in Sec. III. ${ }^{30}$

Several comments are in order. (1) It is obvious that both impurity doping and irradiation (or implantation) can be used to study the disorder effects in superconductors and metals. In particular, compensation of the $T_{c}$ reduction caused by magnetic impurities has been observed as a consequence of both radiation damage and ordinary impurity doping. ${ }^{31-34}$ This compensation phenomenon has been predicted by Kim and Overhauser. ${ }^{35,36}$ Recently, it has also been observed that impurity doping and/or ion-beam-induced damage in high- $T_{c}$ superconductors cause a metal-insulator transition and thereby suppress $T_{c} \cdot{ }^{37-40}$ These reductions may also be understood by the weak localization effect on superconductors. The only difference is the strong renormalization of the impurity potential due to the strong electron-electron interac- 
TABLE I. Irradiation effects on $A 15$ compounds, Chevrel phases, and $\mathrm{NbSe}_{2}$.

\begin{tabular}{|c|c|c|c|c|c|}
\hline Sample & Irradiating particle & $T_{c 0}$ & $\Delta T_{c}$ & Maximum fluence & Reference \\
\hline \multirow[t]{2}{*}{$\mathrm{Nb}_{3} \mathrm{Ge}$} & $\alpha$ particle & $\sim 20 \mathrm{~K}$ & $\sim 8 \mathrm{~K}$ & $10^{17} \alpha / \mathrm{cm}^{2}$ & 8 \\
\hline & Neutron & $\sim 20 \mathrm{~K}$ & $\sim 16 \mathrm{~K}$ & $5 \times 10^{19}$ neutron $/ \mathrm{cm}^{2}$ & 3 \\
\hline \multirow[t]{3}{*}{$\mathrm{Nb}_{3} \mathrm{Sn}$} & $\alpha$ particle & $\sim 18 \mathrm{~K}$ & $\sim 15 \mathrm{~K}$ & $7 \times 10^{17} \alpha / \mathrm{cm}^{2}$ & 11 \\
\hline & Neutron & $\sim 18 \mathrm{~K}$ & $\sim 7 \mathrm{~K}$ & $2 \times 10^{19}$ neutron $/ \mathrm{cm}^{2}$ & 5 \\
\hline & Electron & $\sim 17.8 \mathrm{~K}$ & $\sim 3.8 \mathrm{~K}$ & $4 \times 10^{20}$ electron $/ \mathrm{cm}^{2}$ & 20 \\
\hline $\mathrm{Nb}_{3} \mathrm{Al}$ & Neutron & $\sim 18 \mathrm{~K}$ & $\sim 14 \mathrm{~K}$ & $5 \times 10^{19}$ neutron $/ \mathrm{cm}^{2}$ & 6 \\
\hline $\mathrm{Nb}_{3} \mathrm{Pt}$ & Neutron & $\sim 10.6 \mathrm{~K}$ & $\sim 8.4 \mathrm{~K}$ & $3 \times 10^{19}$ neutron $/ \mathrm{cm}^{2}$ & 24 \\
\hline \multirow[t]{2}{*}{$\mathrm{V}_{3} \mathrm{Si}$} & $\alpha$ particle & $16.8 \mathrm{~K}$ & $\sim 14.5 \mathrm{~K}$ & $7 \times 10^{17} \alpha / \mathrm{cm}^{2}$ & 11 \\
\hline & Neutron & $\sim 16.5 \mathrm{~K}$ & $\sim 13.5 \mathrm{~K}$ & $2.5 \times 10^{19}$ neutron $/ \mathrm{cm}^{2}$ & 3 \\
\hline $\mathrm{V}_{3} \mathrm{Ge}$ & $\alpha$ particle & $6.5 \mathrm{~K}$ & $\sim 5.5 \mathrm{~K}$ & $5 \times 10^{17} \alpha / \mathrm{cm}^{2}$ & 11 \\
\hline $\mathrm{PbMo}_{6} \mathrm{~S}_{8}$ & Neutron & $12.8 \mathrm{~K}$ & $\sim 8.6 \mathrm{~K}$ & $1 \times 10^{19}$ neutron $/ \mathrm{cm}^{2}$ & 3 \\
\hline $\mathrm{PbMo}_{6} \mathrm{~S}_{7}$ & Neutron & & $61 \%$ & $1.5 \times 10^{19}$ neutron $/ \mathrm{cm}^{2}$ & 3 \\
\hline $\mathrm{SnMo}_{5} \mathrm{~S}_{8}$ & Neutron & & $51 \%$ & $1.5 \times 10^{19}$ neutron $/ \mathrm{cm}^{2}$ & 3 \\
\hline $2 \mathrm{H}-\mathrm{NbSe}_{2}$ & Neutron & & $\sim 50 \%$ & $3 \times 10^{18}$ neutron $/ \mathrm{cm}^{2}$ & 3 \\
\hline
\end{tabular}

tion in high- $T_{c}$ superconductors. (2) Although Anderson's theorem suggests no change of the electron-phonon interaction due to the very strong disorder, there is overwhelming experimental evidence for a decrease of the electron-phonon interaction in the strongly disordered samples and in the high-fluence regime. For instance, tunneling, ${ }^{16-18}$ specific heat, ${ }^{41} \mathrm{x}$-ray photoemission spectroscopy (XPS), ${ }^{42}$ loss of thermal electrical resistivity, ${ }^{4}$ and the correlation of $T_{c}$ and the resistance ratio ${ }^{4,8,9,11}$ reveal the decrease of the electronphonon interaction when the electrons are weakly localized. Weak localization leads to a decrease of the amplitude of the electron wave function. As a result, the phonon-mediated matrix elements are also decreasing. ${ }^{28,29}$ (3) Tunneling data do not show any enhancement of the Coulomb repulsion. ${ }^{16-18}$ In addition, the loss of the thermal electrical resistivity with decreasing $T_{c}$ and the universal correlation between $T_{c}$ and resistance ratio cannot be explained in terms of the increase of the Coulomb interaction. (4) Irradiation also leads to strong $T_{c}$ reductions in Chevrel phase materials, such as $\mathrm{PbMo}_{6} \mathrm{~S}_{8}, \mathrm{PbMo}_{6} \mathrm{~S}_{7}$, and $\mathrm{SnMo}_{5} \mathrm{~S}_{8}, 3,43,44$ and $\mathrm{NbSe}_{2},{ }^{43}$ at fluences above $\sim 10^{18}$ neutron $/ \mathrm{cm}^{2}$. These materials are more radiation sensitive than $A 15$ compounds. It is clear that the origin of the strong $T_{c}$ reduction is not related to the microscopic details of the disorder, but related to the universal nature of the electronic state in the irradiated samples.

In Sec. II, we briefly review the experimental results of the radiation damage effects on $A 15$ compounds. The universal large reduction of $T_{c}$, the accompanying decrease of thermal electrical resistivity, and the correlation of $T_{c}$ and the resistance ratio will be emphasized. In Sec. III, the weak localization correction on the phonon-mediated interaction is derived. The resulting $T_{c}$ decrease will be compared with experiments in Sec. IV.

\section{RADIATION EFFECTS IN $\boldsymbol{A} 15$ COMPOUND SUPERCONDUCTORS: UNIVERSAL $T_{c}$ REDUCTION AND RESISTANCE RATIO}

Several $A 15$ compounds have been investigated, including $\quad \mathrm{Nb}_{3} \mathrm{Sn},{ }^{5-7,11-13,20}, \quad \mathrm{Nb}_{3} \mathrm{Al},{ }^{6,7,10}, \quad \mathrm{Nb}_{3} \mathrm{Ge},{ }^{4,6-8,11,13}$,
$\mathrm{Nb}_{3} \mathrm{Ga}^{6,7}, \mathrm{Nb}_{3} \mathrm{Pt},{ }^{24}, \mathrm{~V}_{3} \mathrm{Si}^{4,7,11}$, and $\mathrm{V}_{3} \mathrm{Ge}^{4,11}$. Both highenergy neutron ${ }^{5,6,10}$ and other energetic charged particles, such as protons, ${ }^{14} \alpha$ particles, ${ }^{4,8,11,13}$ oxygens, ${ }^{12}$ and electrons, ${ }^{20}$ were used to irradiate a variety of $A 15$ compounds. Table I summarizes the experimental results of the irradiation effects on $A 15$ compound superconductors, Chevrel phases, and $\mathrm{NbSe}_{2}$. Note that the large $T_{c}$ reductions are found in not only $A 15$ compound superconductors but also Chevrel phase superconductors, ternary superconductors, and $\mathrm{NbSe}_{2}$, implying the universality of the phenomenon.

The response of the superconducting properties of $A 15$ compounds to irradiation can be classified into behavior at low fluences and at higher fluences. In the low-fluence regime, little or no change in $T_{c}$ occurs, while universal large reductions of $T_{c}$ are observed for higher fluences. We focus on the universal $T_{c}$ reduction in this paper. The boundary between the two regimes depends on the irradiating particles, since heavy ions give rise to more severe radiation damage. For instance, the low-fluence regime corresponds to neutron fluence $<\sim 10^{18}$ neutron $/ \mathrm{cm}^{2}$ and to ${ }^{4} \mathrm{He}$ fluence $<\sim 10^{15}{ }^{4} \mathrm{He} / \mathrm{cm}^{2}$. For much more higher fluences the saturation of $T_{c}$ is often found. It is noteworthy that the saturated $T_{c}$ state is accompanied by a saturated value of the residual resistivity $\rho_{0}{ }^{4}$ Accordingly, classification based on the residual resistivity (not the fluence) may be more appropriate. In terms of the residual resistivity, the low-fluence regime corresponds to $\rho_{0}<\sim 10 \mu \Omega \mathrm{cm}$ irrespective of the irradiating particles. The saturations of $T_{c}$ and the residual resistivity are easily understood in this classification scheme.

From Table I, it is clear that the universal $T_{c}$ reduction is not crucially dependent on any specific irradiating particle, any specific material, and any specific defect. Many physicists noticed that the universal $T_{c}$ reduction is governed by the total residual resistivity $\rho_{0}$ due to the radiation damage and the inherent damage present in the sample. ${ }^{45,4,8,9,11}$ Furthermore, the close relation between the $T_{c}$ decrease and resistance ratio was established in $\mathrm{Nb}-\mathrm{Ge}, \mathrm{V}_{3} \mathrm{Si}$, and $\mathrm{V}_{3} \mathrm{Ge}^{4,8,9,11}$ The relation was also noticed in $\mathrm{Nb}-\mathrm{O}$ solid solutions. ${ }^{46}$ Testardi and co-workers ${ }^{4,8,9,11}$ reported that the correlation of $T_{c}$ and the resistance ratio is independent of all 
sputtering conditions, film thickness, composition, and radiation damage. This result implys that defects produced during irradiation are similar in their effect on $T_{c}$ to those produced during the film growth process. Consequently, the correlation of $T_{c}$ and the resistance ratio is also universal. Until the resistance ratio is about $5, T_{c}$ does not change much. When it is smaller than 2, $T_{c}$ drops quickly. Finally superconductivity disappears if the resistance ratio is around 1. Testardi et al. ${ }^{4}$ also found that decreasing $T_{c}$ is accompanied by the loss of the thermal electrical resistivity (electron-phonon interaction), which indicates the significant role of defects in both superconducting and normal-state behavior. This finding, consistent with the correlation of $T_{c}$ and the resistance ratio, predicts the complete destruction of superconductivity for a resistance ratio less than 1 because of the complete loss of the electron-phonon interaction.

Other evidence for the decrease of a electron-phonon interaction in the high fluence regime is the following: (1) By channeling measurements in single-crystal $\mathrm{V}_{3} \mathrm{Si}$, Testardi et $a l^{47}$ also found that radiation damage leads to large increase of the resistivity and a reduction of the electronphonon interaction. (2) Viswanathan and Caton ${ }^{48}$ reported the correlation of $T_{c}$ and the residual resistivity in neutronirradiated $\mathrm{V}_{3}$ Si. (3) Tsuei, Molnar, and Coey ${ }^{41}$ did a comparative study of the superconducting and normal-state properties of the amorphous and crystalline phases of $\mathrm{Nb}_{3} \mathrm{Ge}$. They found that the drastic reduction of $T_{c}$ is due to the changes in the strength of the electron-phonon interaction. Pollak, Tsuei, and Johnson ${ }^{42}$ did an XPS study of the crystalline and amorphous phases of $\mathrm{Nb}_{3} \mathrm{Ge}$ and found that the crystalline phase has a higher $T_{c}$ because of the enhancement of the electron-phonon coupling.

The response of the critical current $I_{c}$ also depends on the fluence. For the low-fluence regime, $I_{c}$ decreases first with fluence, and then increases with increasing fluence, implying the importance of the flux-pinning mechanism. ${ }^{4,50}$ For higher fluences, the universal reduction of $T_{c}$ drives down $I_{c} \cdot{ }^{5,51,52}$ The $I_{c}$ drop is field independent. ${ }^{53}$

\section{WEAK LOCALIZATION CORRECTION TO THE PHONON-MEDIATED INTERACTION}

In the presence of an impurity potential $U_{0}$, the Hamiltonian is given by

$$
\begin{aligned}
H= & \int d \mathbf{r} \sum_{\alpha} \Psi_{\alpha}^{\dagger}(\mathbf{r})\left[\frac{p^{2}}{2 m}+U_{0}(\mathbf{r})\right] \Psi_{\alpha}(\mathbf{r}) \\
& -V \int \Psi_{\uparrow}^{\dagger}(\mathbf{r}) \Psi_{\downarrow}^{\dagger}(\mathbf{r}) \Psi_{\downarrow}(\mathbf{r}) \Psi_{\uparrow}(\mathbf{r}) d \mathbf{r},
\end{aligned}
$$

where $\Psi^{\dagger}(\mathbf{r})$ and $\Psi(\mathbf{r})$ are creation and annihilation operators for electrons. In terms of the exact scattered states $\psi_{n}(\mathbf{r})$, we expand the field operator $\Psi_{\alpha}(\mathbf{r})$ as

$$
\Psi_{\alpha}(\mathbf{r})=\sum_{n} \psi_{n}(\mathbf{r}) c_{n \alpha}
$$

where $c_{n \alpha}$ is a destruction operator of the electron. Upon substituting Eq. (2) into Eq. (1), we find

$$
H=\sum_{n} \epsilon_{n} c_{n \alpha}^{\dagger} c_{n \alpha}-\sum_{n n^{\prime}, n \neq n^{\prime}} V_{n n^{\prime}} c_{n^{\prime} \uparrow}^{\dagger} c_{n^{\prime} \downarrow}^{\dagger} c_{n \downarrow}^{-} c_{n \uparrow},
$$

where

$$
V_{n n^{\prime}}=V \int \psi_{n^{\prime}}^{*}(\mathbf{r}) \psi_{n^{\prime}}^{*}(\mathbf{r}) \psi_{n}^{-}(\mathbf{r}) \psi_{n}(\mathbf{r}) d \mathbf{r} .
$$

Here $\epsilon_{n}$ is the normal-state eigenenergy and $\bar{n}$ denotes the time-reversed partner of the scattered state $n$. Equation (4) was first obtained by Ma and Lee. ${ }^{54}$

\section{A. Andersons' theorem}

By a unitary transformation between the scattered states and the plane-wave states,

$$
\psi_{n \alpha}=\sum_{\vec{k}} \phi_{\vec{k} \alpha}\langle\vec{k} \mid n\rangle
$$

Equation (4) can be rewritten as ${ }^{26}$

$$
V_{n n^{\prime}}=V \sum_{\vec{k}, \vec{k}^{\prime} \vec{q}}\left\langle-\vec{k}^{\prime} \mid n\right\rangle\langle\vec{k} \mid n\rangle^{*}\left\langle\vec{k}-\vec{q} \mid n^{\prime}\right\rangle\left\langle-\vec{k}^{\prime}-\vec{q} \mid n^{\prime}\right\rangle^{*} .
$$

Anderson ${ }^{27}$ assumed that the transformed BCS part in Eq. (6) plays a much more important role and each individual matrix element of the remaining interaction is so small as to be safely disregarded. Then the normalization condition of the scattered states leads to

$$
\begin{aligned}
V_{n n^{\prime}} & \cong V_{n n^{\prime}}^{B C S} \\
& =V \sum_{\vec{k}, \vec{k}^{\prime}}|\langle\vec{k} \mid n\rangle|^{2}\left|\left\langle n^{\prime} \mid \vec{k}^{\prime}\right\rangle\right|^{2}=V,
\end{aligned}
$$

which is the essence of Anderson's theorem.

However, the remaining term

$$
\begin{aligned}
V_{n n^{\prime}}^{n o n-B C S}= & V \sum_{\vec{k} \neq-\vec{k}^{\prime}, \vec{k}^{\prime} \vec{q}}\left\langle-\vec{k}^{\prime} \mid n\right\rangle\langle\vec{k} \mid n\rangle^{*}\left\langle\vec{k}-\vec{q} \mid n^{\prime}\right\rangle \\
& \times\left\langle-\vec{k}^{\prime}-\vec{q} \mid n^{\prime}\right\rangle^{*}
\end{aligned}
$$

cannot always be ignored. As Anderson suggested, the above term is indeed negligible in low-fluence regime, where residual resistivity is smaller than $10 \mu \Omega \mathrm{cm}$. In fact, the lowfluence regime corresponds to the dirty limit where $1 / k_{F} l$ $<0.1 . k_{F}$ and $l$ denote the Fermi wave vector and mean free path, respectively, whereas for higher fluences, the remaining term contributes significantly. In this regime, the electron wave functions are weakly localized. Note that weak localization yields the well-known weak localization correction to the conductivity.

Now we calculate $V_{n n^{\prime}}$ including both BCS and non-BCS terms. In order to do this, we use Eq. (4) [not Eq. (6)] which is more physically transparent. In the dirty limit, the exact eigenstates $\psi_{n}(\mathbf{r})$ can be approximated by the incoherent superpositions of plane-wave states suggested by Thouless, ${ }^{55}$ which leads to the Boltzmann conductivity. The wave function, with energy $\hbar^{2} k_{n}^{2} / 2 m$, is written as 


$$
\psi_{n}(\mathbf{r})=\sum_{\vec{k}} a_{\vec{k}}^{n} e^{i \vec{k} \cdot \mathbf{r}} .
$$

The amplitudes $a_{\vec{k}}^{n}$ are assumed to be independent normally distributed random variables with variance

$$
\overline{a_{\vec{k}}^{n} * a_{\overrightarrow{k^{\prime}}}^{n^{\prime}}} \cong \delta_{n n^{\prime}} \delta_{\vec{k} k^{\prime}} \frac{\pi}{\Omega k_{n}^{2} l} \frac{1}{\left(k-k_{n}\right)^{2}+1 / 4 l^{2}},
$$

for large $k_{n} l$. Here $\Omega$ denotes the volume of the system.

Inserting Eq. (9) into Eq. (4) we obtain

$$
\begin{aligned}
V_{n n^{\prime}} & =V \int\left|\psi_{n^{\prime}}(\mathbf{r})\right|^{2}\left|\psi_{n}(\mathbf{r})\right|^{2} d \mathbf{r} \\
& =V \int \sum_{\vec{k}, q} a_{\vec{k}}^{n} a_{\vec{q}}^{n} * e^{i \vec{k} \cdot \mathbf{r}} e^{-i \vec{q} \cdot \mathbf{r}} \sum_{\vec{k}^{\prime}, q^{\prime}} a_{\vec{k}^{\prime}}^{n^{\prime}} a_{\vec{q}^{\prime}}^{n^{\prime}} * e^{i \vec{k}^{\prime} \cdot \mathbf{r}} e^{-i \vec{q}^{\prime} \cdot \mathbf{r}} d \mathbf{r} \\
& \cong V \int \sum_{\vec{k}} \overline{\left|a_{\vec{k}}^{n}\right|^{2}} \sum_{\vec{k}^{\prime}} \overline{\left|a_{\vec{k}^{\prime}}^{n^{\prime}}\right|^{2}} d \mathbf{r}=V .
\end{aligned}
$$

(Here we assume $\Omega=1$ for the phonon-mediated matrix element, in accordance with the usual notation. ${ }^{27,54}$ ) We have made use of Eq. (10) which eliminated the non-BCS cross terms since $n$ and $n^{\prime}$ are different ${ }^{54}$ and are not dummy indices. As $V_{k k}$ does not contribute to superconductivity in homogeneous systems, ${ }^{56} V_{n n}$ does not contribute to superconductivity in disordered systems. ${ }^{54}$ Here $V_{k k}$ is the (forbidden) diagonal term in the BCS reduced interaction. As a result, Anderson's theorem is proved for large $k_{n} l$ under this assumption.

\section{B. Weak localization correction}

For the high-fluence regime, we may use the weakly localized wave functions suggested by Kaveh and Mott. ${ }^{57,58}$ For three dimensions, the weakly localized wave functions consist of power-law and extended wave functions,

$$
\psi_{\vec{k}}(\mathbf{r})=A e^{i \vec{k} \cdot \mathbf{r}}+B \frac{e^{i k r}}{r^{2}},
$$

where

$$
A^{2}=1-4 \pi B^{2}\left(\frac{1}{l}-\frac{1}{L}\right), \quad B^{2}=\frac{3}{8 \pi} \frac{1}{k_{F}^{2} l} .
$$

$L$ denotes inelastic diffusion length. We then write an eigenstate $\psi_{n}$ as

$$
\psi_{n}(\mathbf{r})=\sum_{\vec{k}} a_{\vec{k}}^{n} \psi_{\vec{k}}(\mathbf{r})=\sum_{\vec{k}} a_{\vec{k}}^{n}\left(A e^{i \vec{k} \cdot \mathbf{r}}+B \frac{e^{i k r}}{r^{2}}\right) .
$$

Comparing to Thouless' wave function, Eq. (9), Kaveh and Mott's wave function includes the power-law component which originated from the diffusive motion of the electrons. While Thouless' wave function corresponds to the Green's function in the self-consistent Born approximation, ${ }^{59,60}$ Kaveh and Mott's wave function corresponds to the Green's function which includes both the Born scattering and the coherent backscattering due to impurities. ${ }^{61,58}$ Since the power-law wave function $1 / r^{2}$ does not contribute to the current, the conductivity is reduced as

$$
\sigma^{3 d}=\sigma_{B} A^{4}=\sigma_{B}\left[1-\frac{3}{\left(k_{F} l\right)^{2}}\left(1-\frac{l}{L}\right)\right] .
$$

A similar situation occurs in the phonon-mediated interaction. The power-law component does not contribute to the phonon-mediated matrix element either. The reason is the following: since the power-law component peaks at some point, its contribution to the bound state of Cooper pairs far from the point is almost negligible. This is analogous to the insensitivity of the localized (bound) state with the change of the boundary conditions. ${ }^{62}$ Accordingly, substitution of Eq. (14) into Eq. (4) leads to the weak localization correction to the phonon-mediated interaction,

$$
\begin{aligned}
V_{n n^{\prime}} & =V \int \sum_{\vec{k}} \overline{\left|a_{\vec{k}}^{n}\right|^{2}} \sum_{\vec{k}^{\prime}} \overline{\left|a_{\vec{k}^{\prime}}^{n^{\prime}}\right|^{2}}\left|\psi_{\vec{k}}(\mathbf{r})\right|^{2}\left|\psi_{\vec{k}^{\prime}}(\mathbf{r})\right|^{2} d \mathbf{r} \\
& =V \int\left|\psi_{\vec{k}}(\mathbf{r})\right|^{2}\left|\psi_{\vec{k}^{\prime}}(\mathbf{r})\right|^{2} d \mathbf{r} \\
& \cong V A^{4}=V\left[1-\frac{3}{\left(k_{F} l\right)^{2}}\left(1-\frac{l}{L}\right)\right] .
\end{aligned}
$$

We have made use of the fact that Eq. (16) does not depend on $\vec{k}$ or $\vec{k}^{\prime}$.

We can also derive the weak localization correction term in Eq. (17) without using Eqs. (12)-(14) based on the diffusive density correlation for the eigenstates. In order to do this, it is important to note that the matrix element $V_{n n^{\prime}}$ denotes the correlation function between two eigenstates $\psi_{n}$ and $\psi_{n^{\prime}}$, as is clear from the expression ${ }^{63}$

$$
V_{n n^{\prime}}=V \int\left|\psi_{n}(\mathbf{r})\right|^{2}\left|\psi_{n^{\prime}}(\mathbf{r})\right|^{2} d \mathbf{r}=\frac{V}{\Omega} \sum_{\vec{q}}\left|\left\langle\psi_{n}\left|e^{i \vec{q} \cdot \mathbf{r}}\right| \psi_{n^{\prime}}\right\rangle\right|^{2}
$$

We have evaluated the sum over $\vec{q}$. For quantum diffusion of electrons, it was shown ${ }^{63-66}$ that

$$
\left|\left\langle\psi_{n}\left|e^{i \vec{q} \cdot \mathbf{r}}\right| \psi_{n^{\prime}}\right\rangle\right|_{A V}^{2}=\frac{1}{2 \pi \hbar N_{0}\left(E_{F}\right)} \frac{D \vec{q}^{2}}{\left(D \vec{q}^{2}\right)^{2}+\left(\epsilon_{n}-\epsilon_{n^{\prime}}\right)^{2} / \hbar^{2}},
$$

where $A V$ means the average over all states, and $N_{0}\left(E_{F}\right)$ and $D$ are the density of states and the diffusion constant, respectively. This quantity is proportional to the spectral function for the density correlation function, $A(q, \omega)$, which is defined as ${ }^{66}$

$$
A(\vec{q}, \omega)=\int_{-\infty}^{\infty} d t d \mathbf{r} d \mathbf{r}^{\prime} e^{i \omega t} e^{i \vec{q} \cdot\left(\mathbf{r}-\mathbf{r}^{\prime}\right)}\left\langle\left[\rho\left(\mathbf{r}^{\prime}, t\right), \rho(\mathbf{r}, 0)\right]\right\rangle,
$$

where $\rho$ is the density operator. In Eq. (19) we may assume $\epsilon_{n}=\epsilon_{n^{\prime}}$, since we are interested in states very near the Fermi energy. 
Note also that in the presence of impurities, the correlation function has a free-particle form for $t<\tau$ (scattering time) and a diffusive form for $t>\tau .{ }^{25}$ As a result, for $t>\tau$ (or $r>l$ ), one finds ${ }^{63}$

$$
\begin{aligned}
R & =\int_{t>\tau}\left|\psi_{n}(\mathbf{r})\right|^{2}\left|\psi_{n^{\prime}}(\mathbf{r})\right|^{2} d \mathbf{r}=\frac{1}{\Omega} \sum_{\vec{q}}\left|\left\langle\psi_{n}\left|e^{i \vec{q} \cdot \mathbf{r}}\right| \psi_{n^{\prime}}\right\rangle\right|_{A V}^{2} \\
& =\frac{1}{\Omega} \sum_{\pi / L<\vec{q}<\pi / l} \frac{1}{2 \pi \hbar N_{0}\left(E_{F}\right) D \vec{q}^{2}} \\
& =\frac{3}{2\left(k_{F} l\right)^{2}}\left(1-\frac{l}{L}\right) .
\end{aligned}
$$

In Eq. (21), the lower limit is $\sim \pi / L$, the upper limit being $\sim \pi / l$, corresponding to the diffusive motion of the electron in real space. Note that $N_{0}\left(E_{F}\right)=m k_{F} / 2 \pi^{2} \hbar^{2}$ and $D$ $=(1 / 3) v_{F} l$, whereas the contribution from the free-particlelike density correlation is

$$
\begin{aligned}
V_{n n^{\prime}} & =V \int_{t<\tau}\left|\psi_{n}(\mathbf{r})\right|^{2}\left|\psi_{n^{\prime}}(\mathbf{r})\right|^{2} d \mathbf{r} \cong V A^{4} \\
& =V\left[1-\frac{3}{\left(k_{F} l\right)^{2}}\left(1-\frac{l}{L}\right)\right],
\end{aligned}
$$

with $A^{2}=1-R{ }^{63}$ Since the phonon-mediated interaction is retarded for $t_{r e t} \sim 1 / \omega_{D}$, only the free-particle-like density correlation contributes to the phonon-mediated matrix element. This leads to the same weak localization correction to both the conductivity and the phonon-mediated matrix element. Here $\omega_{D}$ is the Debye frequency.

The BCS $T_{c}$ equation is, now,

$$
T_{c}=1.13 \omega_{D} e^{-1 / \lambda_{e f f}}
$$

where

$$
\lambda_{e f f}=N_{0} V\left[1-\frac{3}{\left(k_{F} l\right)^{2}}\left(1-\frac{l}{L}\right)\right] .
$$

The initial change in $T_{c}$ relative to $T_{c 0}$ (for pure metal) is then

$$
\begin{aligned}
\frac{\Delta T_{c}}{T_{c 0}} & \cong \frac{1}{\lambda} \frac{3}{\left(k_{F} l\right)^{2}}\left(1-\frac{l}{L}\right) \\
& \propto \rho_{0}^{2},
\end{aligned}
$$

where the BCS $\lambda$ is $N_{0} V$. This result is in good agreement with experiments. ${ }^{13,67-69}$

\section{Strong-coupling theory}

In the strong-coupling theory, ${ }^{70,71}$ the electron-phonon coupling constant is defined by ${ }^{71}$

$$
\lambda=2 \int \frac{\alpha^{2}(\omega) F(\omega)}{\omega} d \omega
$$

$$
=N_{0} \frac{\left\langle I^{2}\right\rangle}{M\left\langle\omega^{2}\right\rangle} .
$$

Here $F(\omega)$ is the phonon density of states and $M$ is the ionic mass. $\left\langle I^{2}\right\rangle$ and $\left\langle\omega^{2}\right\rangle$ are the average over the Fermi surface of the square of the electronic matrix element and the phonon frequency. ${ }^{71}$ For a homogeneous system with the Einstein model, it is written as

$$
\lambda_{0}=N_{0} \frac{I_{0}^{2}}{M \omega_{D}^{2}},
$$

where $I_{0}$ is the electronic matrix element for the plane-wave states and $\omega_{D}$ denotes the Einstein phonon frequency. In BCS theory, Eq. (4) leads to the BCS coupling constant

$$
\lambda_{B C S}=N_{0} V, \quad \text { BCS theory. }
$$

Comparing Eqs. (29) and (30), we get

$$
V=\frac{I_{0}^{2}}{M \omega_{D}^{2}} .
$$

In general, using the equivalent electron-electron potential in the electron-phonon problem, ${ }^{72,73}$

$$
V\left(x-x^{\prime}\right) \rightarrow \frac{I_{0}^{2}}{M \omega_{D}^{2}} D\left(x-x^{\prime}\right),
$$

with $x=(\mathbf{r}, t)$, the Fröhlich interaction at finite temperatures for an Einstein model may be obtained by

$$
\begin{aligned}
V_{\vec{k} \vec{k}^{\prime}}\left(\omega, \omega^{\prime}\right)= & \frac{I_{0}^{2}}{M \omega_{D}^{2}} \iint d \mathbf{r} d \mathbf{r}^{\prime} \phi_{\vec{k}^{\prime}}^{*}(\mathbf{r}) \phi_{-\vec{k}^{\prime}}^{*}\left(\mathbf{r}^{\prime}\right) D\left(\mathbf{r}-\mathbf{r}^{\prime}, \omega\right. \\
& \left.-\omega^{\prime}\right) \phi_{-\vec{k}^{\prime}}\left(\mathbf{r}^{\prime}\right) \phi_{\vec{k}}(\mathbf{r}) \\
= & \frac{I_{0}^{2}}{M \omega_{D}^{2}} \int\left|\phi_{\vec{k}^{\prime}}(\mathbf{r})\right|^{2}\left|\phi_{\vec{k}}(\mathbf{r})\right|^{2} d \mathbf{r} \frac{\omega_{D}^{2}}{\omega_{D}^{2}+\left(\omega-\omega^{\prime}\right)^{2}} \\
= & V_{\vec{k} \vec{k}^{\prime}} \frac{\omega_{D}^{2}}{\omega_{D}^{2}+\left(\omega-\omega^{\prime}\right)^{2}},
\end{aligned}
$$

where $^{73}$

$$
\begin{aligned}
D\left(\mathbf{r}-\mathbf{r}^{\prime}, \omega-\omega^{\prime}\right) & =\frac{1}{\Omega} \sum_{\vec{q}} \frac{\omega_{D}^{2}}{\left(\omega-\omega^{\prime}\right)^{2}+\omega_{D}^{2}} e^{i \vec{q} \cdot\left(\mathbf{r}-\mathbf{r}^{\prime}\right)} \\
& =\frac{\omega_{D}^{2}}{\left(\omega-\omega^{\prime}\right)^{2}+\omega_{D}^{2}} \delta\left(\mathbf{r}-\mathbf{r}^{\prime}\right) .
\end{aligned}
$$

Here $\omega$ means the Matsubara frequency and $\phi_{\vec{k}}$ denotes the plane-wave state. Note that the spatial part of the phonon Green's function $D\left(\mathbf{r}-\mathbf{r}^{\prime}, \omega-\omega^{\prime}\right)$ becomes the Dirac delta function, since the phonon frequency does not depend on the momentum. Consequently, Eq. (33) leads to the coupling constant 


$$
\lambda_{0}=N_{0}\left\langle V_{\vec{k} \vec{k}^{\prime}}(0,0)\right\rangle=N_{0} \frac{I_{0}^{2}}{M \omega_{D}^{2}}
$$

and the strong-coupling gap equation ${ }^{74}$

$$
\begin{aligned}
\Delta(\vec{k}, \omega) & =T \sum_{\omega^{\prime}} \sum_{\vec{k}^{\prime}} V_{\vec{k} \vec{k}^{\prime}}\left(\omega, \omega^{\prime}\right) \frac{\Delta\left(\vec{k}^{\prime}, \omega^{\prime}\right)}{\omega^{\prime 2}+E_{\vec{k}^{\prime}}^{2}\left(\omega^{\prime}\right)} \\
& =T \sum_{\omega^{\prime}} \frac{\omega_{D}^{2}}{\left(\omega-\omega^{\prime}\right)^{2}+\omega_{D}^{2}} \sum_{\vec{k}^{\prime}} V_{\vec{k} \vec{k}^{\prime}} \frac{\Delta\left(\vec{k}^{\prime}, \omega^{\prime}\right)}{\omega^{\prime 2}+E_{\vec{k}^{\prime}}^{2}\left(\omega^{\prime}\right)},
\end{aligned}
$$

where

$$
E_{\vec{k}^{\prime}}\left(\omega^{\prime}\right)=\sqrt{\epsilon_{k^{\prime}}^{2}+\Delta_{k^{\prime}}^{2}\left(\omega^{\prime}\right)} .
$$

In the presence of impurities, weak localization leads to a correction to $\alpha^{2}$ or $\left\langle I^{2}\right\rangle$, [disregarding the changes of $F(\omega)$ and $\left.N_{0}\right]$. From Eq. (32), one finds

$$
\begin{aligned}
V_{n n^{\prime}}\left(\omega, \omega^{\prime}\right)= & \frac{I_{0}^{2}}{M \omega_{D}^{2}} \iint d \mathbf{r} d \mathbf{r}^{\prime} \psi_{n^{\prime}}^{*}(\mathbf{r}) \psi_{n^{\prime}}^{*}\left(\mathbf{r}^{\prime}\right) D\left(\mathbf{r}-\mathbf{r}^{\prime}, \omega\right. \\
& \left.-\omega^{\prime}\right) \psi_{n}^{-}\left(\mathbf{r}^{\prime}\right) \psi_{n}(\mathbf{r}) \\
= & \frac{I_{0}^{2}}{M \omega_{D}^{2}} \int\left|\psi_{n^{\prime}}(\mathbf{r})\right|^{2}\left|\psi_{n}(\mathbf{r})\right|^{2} d \mathbf{r} \frac{\omega_{D}^{2}}{\omega_{D}^{2}+\left(\omega-\omega^{\prime}\right)^{2}} \\
= & V_{n n^{\prime}} \frac{\omega_{D}^{2}}{\omega_{D}^{2}+\left(\omega-\omega^{\prime}\right)^{2}}
\end{aligned}
$$

and

$$
\lambda=N_{0}\left\langle V_{n n^{\prime}}(0,0)\right\rangle=N_{0} \frac{I_{0}^{2}}{M \omega_{D}^{2}}\left\langle\int\left|\psi_{n}(\mathbf{r})\right|^{2}\left|\psi_{n^{\prime}}(\mathbf{r})\right|^{2} d \mathbf{r}\right\rangle,
$$

which agrees with BCS theory:

$$
\lambda_{e f f}=N_{0} V\left\langle\int\left|\psi_{n}(\mathbf{r})\right|^{2}\left|\psi_{n^{\prime}}(\mathbf{r})\right|^{2} d \mathbf{r}\right\rangle .
$$

Therefore, both the weak- and strong-coupling gap equations give basically the same result of the weak localization effect in superconductors.

\section{Resistance ratio}

According to Matthiessen's rule, the resistivity $\rho(T)$ caused by static and thermal disorder is additive, i.e.,

$$
\rho(T)=\rho_{0}+\rho_{p h}(T),
$$

where $\rho_{p h}$ is mostly due to electron-phonon scattering. At high temperatures, the phonon limited electrical resistivity is given by ${ }^{75}$

$$
\rho_{p h}(T)=\frac{4 \pi m k_{B} T}{n e^{2} \hbar} \int \frac{\alpha_{t r}^{2} F(\omega)}{\omega} d \omega,
$$

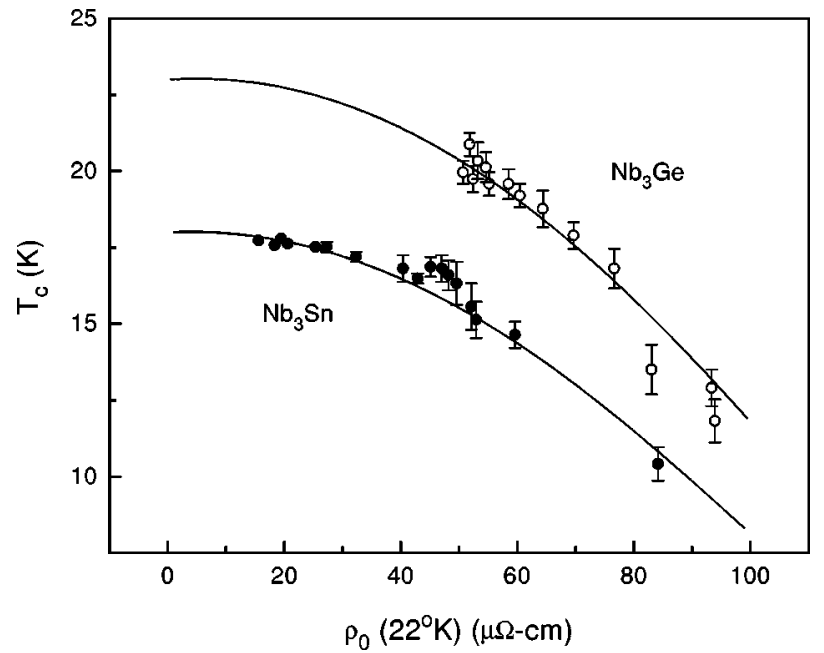

FIG. 1. Calculated $T_{c}$ 's vs residual resistivity $\rho_{0}$ for $\mathrm{Nb}_{3} \mathrm{Ge}$ and $\mathrm{Nb}_{3} \mathrm{Sn}$. Experimental data are due to Wiesmann et al., Ref. 13.

where $\alpha_{t r}$ includes an average of a geometrical factor 1 $-\cos \theta_{\vec{k} k^{\prime}}$. Assuming $\alpha_{t r}^{2} \cong \alpha^{2}$, we obtain

$$
\rho_{p h}(T) \cong \frac{2 \pi m k_{B} T}{n e^{2} \hbar} \lambda_{e f f} \cong \frac{2 \pi m k_{B} T}{n e^{2} \hbar} N_{0} \frac{I_{0}^{2}}{M \omega_{D}^{2}}\left[1-\frac{3}{\left(k_{F} l\right)^{2}}\right] .
$$

Note that decreasing $T_{c}$ is accompanied by the loss of the thermal resistivity $\rho_{p h}(T)$, in good agreement with experiment. ${ }^{4}$ The ternary superconductor $\mathrm{LuRh}_{4} \mathrm{~B}_{4}$ (Ref. 15) also shows the same behavior. The room temperature resistance ratio is then written as

$$
\frac{\rho(300 \mathrm{~K})}{\rho_{0}}=\frac{\rho_{0}+\rho_{p h}(300 \mathrm{~K})}{\rho_{0}} \cong 1+\frac{2 \pi \tau \times 300 \mathrm{~K}}{\hbar} \lambda_{e f f} .
$$

When $\lambda_{\text {eff }}$ goes to zero, the system is not superconducting and resistance ratio becomes 1 , which is in good agreement with experiments. ${ }^{4,8,9,11,46}$ More details will be published elsewhere.

\section{COMPARISON WITH EXPERIMENTS}

Wiesmann et al. ${ }^{13}$ examined the $T_{c}$ change of vapordeposited $\mathrm{Nb}_{3} \mathrm{Ge}$ and $\mathrm{Nb}_{3} \mathrm{Sn}$ as a function of $\alpha$ particle fluence. The $2.5-\mathrm{MeV} \alpha$ particles irradiated the samples, which were held at $30 \mathrm{~K}$. The samples were then cooled, and both $T_{c}$ and the residual resistivity $\rho_{0}$ were measured. Figure 1 shows the dependence of $T_{c}$ on $\rho_{0}$ in $\mathrm{Nb}_{3} \mathrm{Ge}$ and $\mathrm{Nb}_{3} \mathrm{Sn}$. Thin lines are our theoretical results obtained from Eqs. (24) and (25). We find good agreement between theory and experiment. The Debye temperature and $T_{c 0}$ (for the pure sample) are $\omega_{D}=302 \mathrm{~K}, T_{c 0}=23 \mathrm{~K}$ and $\omega_{D}=290 \mathrm{~K}, T_{c 0}$ $=18 \mathrm{~K}$ for $\mathrm{Nb}_{3} \mathrm{Ge}$ and $\mathrm{Nb}_{3} \mathrm{Sn}$, respectively. In the absence of experimental data for the inelastic diffusion length, we used the same value of $L=\sqrt{D \tau_{i}}=\sqrt{l} \times 387 \AA / \mathrm{T}$ for both materials. ${ }^{76}$ Here $\tau_{i}$ means the inelastic scattering time and $T$ denotes temperature/K. We assumed $\tau_{i} \propto T^{-2}$ corresponding to the electron-electron interactions. ${ }^{58,76}$ Since it is very difficult to evaluate $k_{F} l$ accurately, ${ }^{77}$ we assumed that $\rho$ 

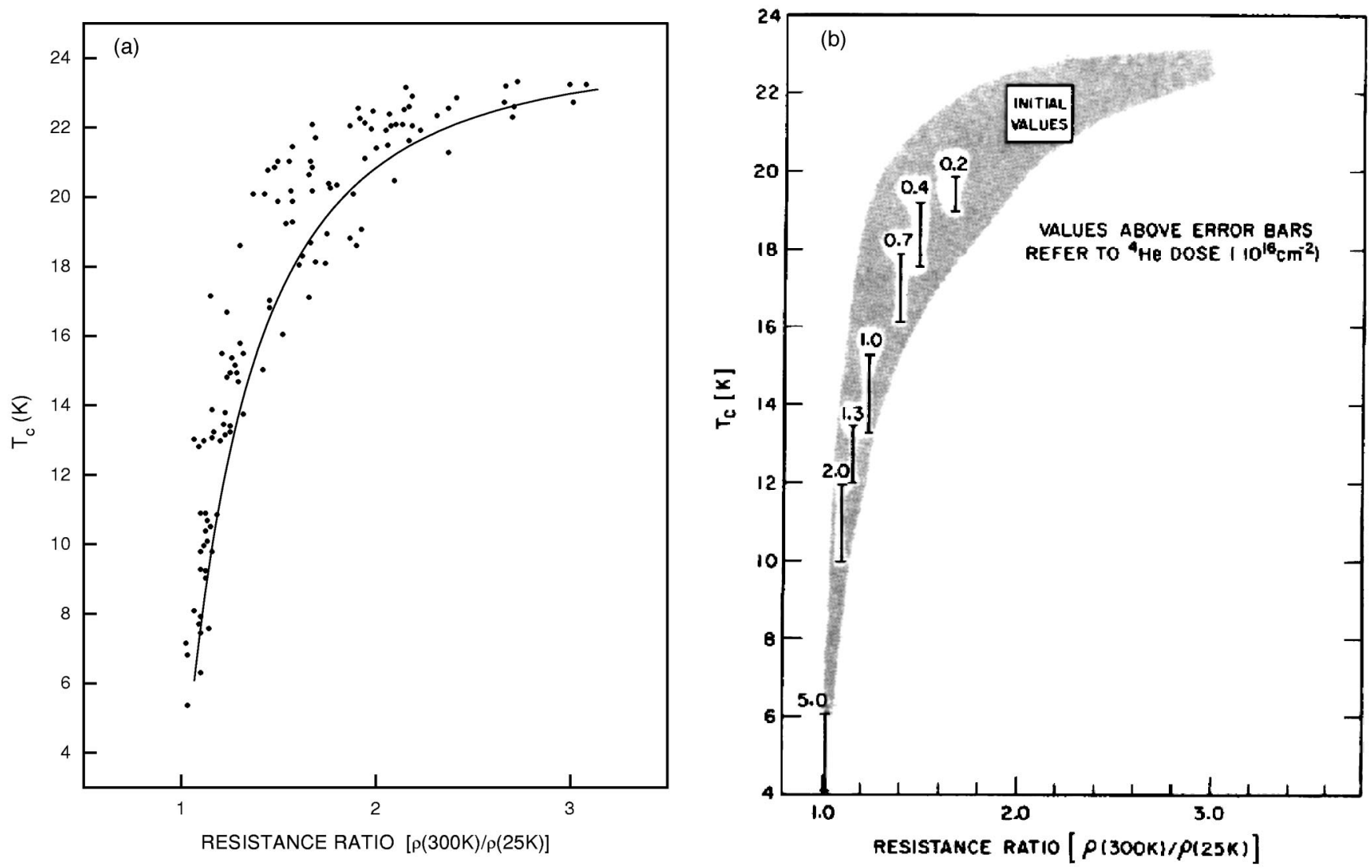

FIG. 2. (a) Calculated $T_{c}$ 's vs resistance ratio for $\mathrm{Nb}-\mathrm{Ge}$. The data points relate to about 130 films made with various sputtering voltage, deposition conditions, film thickness, crystal structure, and chemical composition. Data are from Testardi et al., Ref. 9. (b) $T_{c}$-resistance-ratio correlation band for 130 as-grown films with the values for damaged films superimposed. Data are from Poate et al., Ref. 8 .

$=100 \mu \Omega \mathrm{cm}$ corresponds to $k_{F} l=3.65$ and 3.60 for $\mathrm{Nb}_{3} \mathrm{Ge}$ and $\mathrm{Nb}_{3} \mathrm{Sn}$ with the same value of $k_{F}=0.3 \AA^{-1}$. These values also give a good fitting to the dependence of $T_{c}$ on the residual resistivity in impurity-doped samples. ${ }^{78}$ This is persuasive evidence that the $T_{c}$ behavior is not crucially dependent on any specific defect; rather its behavior is governed by the residual resistivity.

Testardi and co-workers ${ }^{9}$ prepared about $130 \mathrm{Nb}$-Ge films and examined the dependence of $T_{c}$ on resistivity, resistance ratio, chemical composition, and sputtering conditions. The $\mathrm{Nb} / \mathrm{Ge}$ ratios were in the range of $\sim 2.4-5.5$ and film thickness were about 2000-3500 А. Only films which show a width of the superconducting transition less than $\sim 2-3 \mathrm{~K}$ were chosen to ensure the macroscopic homogeneity of the samples. They found a universal correlation of $T_{c}$ and resistance ratio irrespective of all sputtering conditions, composition, and specific nature of the disorder. ${ }^{4,8,9,11,79}$ Figure 2 (a) presents a sampling of $T_{c}$-vs-resistance-ratio data for 130 $\mathrm{Nb}-\mathrm{Ge}$ films by them. The correlation between $T_{c}$ and the resistance ratio is obvious. Resistance ratios less than 1 were generally found in films which are not superconducting, which agrees with theoretical expression, Eq. (44). Our theoretical curve, which was obtained from Eqs. (24), (25), and (44), is also shown in the same figure. We again find good agreement between the theoretical curve and experiment. Since A 15 compounds show deviations from Matthiessen's rule possibly due to saturation, ${ }^{4,80}$ we adjusted $\rho_{p h}(T)$ to fit experimental values ${ }^{4}$ at $300 \mathrm{~K}$ in the following manner: $\rho_{p h}(300 \mathrm{~K}) \cong 90 \mu \Omega \mathrm{cm} \times \lambda_{e f f}\left(1-2.4 / k_{F} l\right) / \lambda_{0}$. We used the same values for $k_{F}, L$, and $\omega_{D}$ as in Fig. 1. But we found that $T_{c 0}=24 \mathrm{~K}$ for pure $\mathrm{Nb}_{3} \mathrm{Ge}$ gives a better fitting, which supports the conjecture that sputtered films may have not yet achieved the highest possible $T_{c}$ 's. ${ }^{9}$

Poate et $a l .{ }^{8}$ irradiated superconducting $\mathrm{Nb}-\mathrm{Ge}$ films by $2-\mathrm{MeV} \alpha$ particles and found a $T_{c}$-resistance correlation similar to that as-grown films. Figure 2(b) shows the correlations both for 130 as-grown films ${ }^{9}$ and for $\alpha$-particle-irradiated films. ${ }^{8}$ They lie nicely within the correlation band. It indicates that the correlation of $T_{c}$ of the resistance ratio is universal irrespective of how disorder is caused, e.g., by irradiation or substitutional alloying. Therefore, our theory provides an explanation of both data. The correlation was also reported in $\mathrm{V}_{3} \mathrm{Si}$ and $\mathrm{V}_{3} \mathrm{Ge}^{4}$

\section{DISCUSSION}

It is clear that the weak localization effect in superconductors caused by impurity doping or radiation damage should be subjected to further experimental study. In particular, since the same weak localization correction term occurs in both the conductivity and the phonon-mediated interactions, comparative study of the normal and superconducting properties of the samples will be beneficial. It is noteworthy that Fiory and Hebard ${ }^{69}$ found that both the conductivity and 
the transition temperature vary as $\left(k_{F} l\right)^{-2}$ for bulk amorphous $\mathrm{InO}_{x}$.

The antilocalization effect of the spin-orbit interaction will provide more insights into the weak localization effect in superconductors. In fact, Miller et al. ${ }^{81}$ found compensation for the $T_{c}$ decrease in highly disordered superconductors by adding impurities with large spin-orbit scattering.

The loss of thermal electrical resistivity $\rho_{p h}(T)$ (electronphonon interaction) with decreasing $T_{c}$ needs more experimental study. In particular, we may consider samples satisfying Matthiessen's rule, where correlation of $T_{c}$ and the resistance ratio is more physically transparent. We propose to investigate the usual low- $T_{c}$ superconductors near the superconductor-insulator transition. ${ }^{82} \mathrm{We}$ expect to find the loss of thermal electrical resistivity as approaching the insulating regime. Unfortunately, no systematic study is available yet. Note that this behavior may provide a means of probing the phononmechanism in exotic superconductors, such as, heavy fermion superconductors, organic superconductors, and high- $T_{c}$ cuprates.

\section{CONCLUSION}

We have considered irradiation effects on $A 15$ superconductors. The universal large reduction of $T_{c}$ and $I_{c}$ due to radiation damage has been explained by the weak localization of electrons. Using the weak localization correction to the phonon-mediated interaction derived from the density correlation function, we calculated $T_{c}$ values which are in good agreement with experimental data. It is shown that weak localization decreases significantly both the electronphonon interaction and the phonon-mediated interaction, and thereby gives rise to the universal correlation of $T_{c}$ and the resistance ratio.

\section{ACKNOWLEDGMENTS}

Y.J.K. is grateful to Professor Yun Kyu Bang and Professor Bilal Tanatar for discussions and encouragement. M.P. thanks the FOPI at the University of Puerto Rico-Humacao for release time.
${ }^{1}$ S. V. Vonsovsky, Y. A. Izyumov, and E. Z. Kurmaev, Superconductivity of Transition Metals (Springer-Verlag, Berlin, 1982), Chap. 9.

${ }^{2}$ C. L. Snead, Jr. and T. Luhman, in Physics of Radiation Effects in Crystals, edited by R. A. Johnson and A. N. Orlov (Elsevier, New York, 1986), Chap. 6.

${ }^{3}$ A. R. Sweedler, C. L. Snead, Jr., and D. E. Cox, in Treatise on Materials Science and Technology, edited by Th. Luhman and D. Dew-Hughes (Academic Press, New York, 1979), Vol. 14, pp. 349-426.

${ }^{4}$ L.R. Testardi, J.M. Poate, and H.J. Levinstein, Phys. Rev. B 15, 2570 (1977).

${ }^{5}$ R. Bett, Cryogenics 14, 361 (1974).

${ }^{6}$ A.R. Sweedler, D.S. Schweitzer, and G.W. Webb, Phys. Rev. Lett. 33, 168 (1974).

${ }^{7}$ A.R. Sweedler, D.E. Cox, and L. Newkirk, J. Electron. Mater. 4, 883 (1975).

${ }^{8}$ J.M. Poate, L.R. Testardi, A.R. Storm, and W.M. Augustyniak, Phys. Rev. Lett. 35, 1290 (1975).

${ }^{9}$ L.R. Testardi, R.L. Meek, J.M. Poate, W.A. Royer, A.R. Storm, and J.H. Wernick, Phys. Rev. B 11, 4304 (1975).

${ }^{10}$ A.R. Sweedler and D.E. Cox, Phys. Rev. B 12, 147 (1975).

${ }^{11}$ J.M. Poate, R.C. Dynes, L.R. Testardi, and R.H. Hammond, Phys. Rev. Lett. 37, 1308 (1976).

${ }^{12}$ G. Ischenko, H. Adrian, S. Klaümunzer, M. Lehmann, P. Müller, H. Neumüller, and W. Szymczak, Phys. Rev. Lett. 39, 43 (1977).

${ }^{13}$ H. Wiesmann, M. Gurvitch, A.K. Ghosh, H. Lutz, K.W. Jones, A.N. Goland, and M. Strongin, J. Low Temp. Phys. 30, 513 (1978).

${ }^{14}$ C.L. Snead, Jr., J. Nucl. Mater. 72, 192 (1978).

${ }^{15}$ R. C. Dynes, J. M. Rowell, and P. H. Schmidt, in Ternary Superconductors, edited by G. K. Shenoy, B. D. Dunlap, and F. Y. Fradin (North-Holland, Amsterdam, 1981), p. 169.

${ }^{16}$ K.E. Kihlstrom, D. Mael, and T.H. Geballe, Phys. Rev. B 29, 150 (1984).

${ }^{17}$ D.A. Rudman and M.R. Beasley, Phys. Rev. B 30, 2590 (1984).
${ }^{18}$ J. Geerk, H. Rietschel, and U. Schneider, Phys. Rev. B 30, 459 (1984).

${ }^{19}$ R.C. Dynes and C.M. Varma, J. Phys. F: Met. Phys. 6, L215 (1976).

${ }^{20}$ M. Gurvitch, A.K. Ghosh, C.L. Snead, Jr., and M. Strongin, Phys. Rev. Lett. 39, 1102 (1977)

${ }^{21}$ H. Wiesmann, M. Gurvitch, A.K. Ghosh, H. Lutz, O.F. Kammerer, and M. Strongin, Phys. Rev. B 17, 122 (1978).

${ }^{22}$ A.K. Ghosh, M. Gurvitch, H. Wiesmann, and M. Strongin, Phys. Rev. B 18, 6116 (1978).

${ }^{23}$ D.F. Farrell and B.S. Chandrasekar, Phys. Rev. Lett. 38, 788 (1977).

${ }^{24}$ A.R. Sweedler, D.E. Cox, and S. Moehlecke, J. Nucl. Mater. 72, 50 (1978).

${ }^{25}$ P.W. Anderson, K.A. Muttalib, and T.V. Ramakrishnan, Phys. Rev. B 28, 117 (1983).

${ }^{26}$ Yong-Jihn Kim and A.W. Overhauser, Phys. Rev. B 47, 8025 (1993).

${ }^{27}$ P.W. Anderson, J. Phys. Chem. Solids 11, 26 (1959).

${ }^{28}$ Yong-Jihn Kim, Mod. Phys. Lett. B 10, 555 (1996).

${ }^{29}$ Yong-Jihn Kim, Int. J. Mod. Phys. B 11, 1731 (1997).

${ }^{30}$ Mi-Ae Park and Yong-Jihn Kim, Bull. Am. Phys. Soc. 44, 1589 (1999).

${ }^{31}$ M.F. Merriam, S.H. Liu, and D.P. Seraphim, Phys. Rev. 136, A17 (1964).

${ }^{32}$ G. Boato, G. Gallinaro, and C. Rizzuto, Phys. Rev. 148, 353 (1966).

${ }^{33}$ A. Hofmann, W. Bauriedl, and P. Ziemann, Z. Phys. B: Condens. Matter 46, 117 (1982).

${ }^{34}$ M. Hitzfeld and G. Heim, Solid State Commun. 29, 93 (1979).

${ }^{35}$ Yong-Jihn Kim and A.W. Overhauser, Phys. Rev. B 49, 15799 (1994).

${ }^{36}$ Mi-Ae Park, M.H. Lee, and Yong-Jihn Kim, Physica C 306, 96 (1998).

${ }^{37}$ J.M. Valles, Jr., A.E. White, K.T. Short, R.C. Dynes, J.P. Garno, A.F. Levi, M. Anzlowar, and K. Baldwin, Phys. Rev. B 39, 11599 (1989). 
${ }^{38}$ Y. Li, G. Xiong, and Z. Gan, Physica C 199, 269 (1992).

${ }^{39}$ Y. Dalichaouch, M.S. Torikachvili, E.A. Early, B.W. Lee, C.L. Seaman, K.N. Yang, H. Zhou, and M.B. Maple, Solid State Commun. 65, 1001 (1988).

${ }^{40}$ J. Fink, N. Nücker, H. Romberg, M. Alexander, M.B. Maple, J.J. Neumeier, and J.W. Allen, Phys. Rev. B 42, 4823 (1990).

${ }^{41}$ C.C. Tsuei, S. von. Molnar, and J.M. Coey, Phys. Rev. Lett. 41, 664 (1978).

${ }^{42}$ R.A. Pollak, C.C. Tsuei, and R.W. Johnson, Solid State Commun. 23, 879 (1977)

${ }^{43}$ A. R. Sweedler et al. (unpublished).

${ }^{44}$ B.S. Brown, J.W. Hafstrom, and T.E. Klippert, J. Appl. Phys. 48, 1759 (1977).

${ }^{45}$ A.K. Ghosh, H. Weismann, M. Gurvitch, H. Lutz, O.F. Kammerer, C.L. Snead, A. Goland, and M. Strongin, J. Nucl. Mater. 72, 70 (1978).

${ }^{46}$ C.C. Koch, J.O. Scarbrough, and D.M. Kroeger, Phys. Rev. B 9 , 888 (1974).

${ }^{47}$ L.R. Testardi, J.M. Poate, W. Weber, W.M. Augustyniak, and J.H. Barret, Phys. Rev. Lett. 39, 716 (1977).

${ }^{48}$ R. Viswanathan and R. Caton, Phys. Rev. B 18, 15 (1978).

${ }^{49}$ B.S. Brown, T.H. Blewitt, D.G. Wozniak, and M. Suenaga, J. Appl. Phys. 46, 5163 (1975).

${ }^{50}$ B.S. Brown, T.H. Blewitt, T.L. Scott, and D.G. Wozniak, J. Appl. Phys. 49, 4144 (1978).

${ }^{51}$ D.G. Schweitzer and D.M. Parkin, Appl. Phys. Lett. 24, 333 (1974).

${ }^{52}$ D.M. Parkin, and D.G. Schweitzer, Nucl. Technol. 22, 108 (1974).

${ }^{53}$ C.L. Snead, Jr. and D.M. Parkin, Nucl. Technol. 29, 264 (1976).

${ }^{54}$ M. Ma and P.A. Lee, Phys. Rev. B 32, 5658 (1985).

${ }^{55}$ D.J. Thouless, Philos. Mag. 32, 877 (1975).

${ }^{56}$ C. Kittel, Quantum Theory of Solids (Wiley, New York, 1963), Eq. (53) in Chap. 8.

${ }^{57}$ M. Kaveh and N.F. Mott, J. Phys. C 14, L177 (1981).

${ }^{58}$ N.F. Mott and M. Kaveh, Adv. Phys. 34, 329 (1985).

${ }^{59}$ A. D. Stone, in Physics of Nanostructures, edited by J. H. Davies and A. R. Long (IOP, Bristol, 1992), p. 65.

${ }^{60}$ P. L. Taylor, Quantum Approach to the Solid State (Prentice-Hall, Englewood Cliffs, NJ, 1970), p. 286.
${ }^{61}$ G. Bergmann, Phys. Rep. 101, 1 (1984).

${ }^{62}$ D.J. Thouless, Phys. Rep., Phys. Lett. 13C, 93 (1974).

${ }^{63}$ M. Kaveh, Philos. Mag. B 51, 453 (1985).

${ }^{64}$ W.L. McMillan, Phys. Rev. B 24, 2739 (1981).

${ }^{65}$ B.L. Altshuler and A.G. Aronov, Zh. Éksp. Teor. Fiz. 77, 2028 (1979) [Sov. Phys. JETP 50, 968 (1979)].

${ }^{66}$ E. Abrahams, P.W. Anderson, P.A. Lee, and T.V. Ramakrishnan, Phys. Rev. B 24, 6783 (1981).

${ }^{67}$ J.P. Orlando, E.J. McNiff, Jr., S. Foner, and M.R. Beasley, Phys. Rev. B 19, 4545 (1979).

${ }^{68}$ S.J. Bending, M.R. Beasley, and C.C. Tsuei, Phys. Rev. B 30, 6342 (1984).

${ }^{69}$ A.T. Fiory and A.F. Hebard, Phys. Rev. Lett. 52, 2057 (1984).

${ }^{70}$ G.M. Eliashberg, Zh. Éksp. Teor. Fiz. 38, 966 (1960) [Sov. Phys. JETP 11, 696 (1960)].

${ }^{71}$ W.L. McMillan, Phys. Rev. 167, 331 (1968).

${ }^{72}$ A. A. Abrikosov, L. P. Gor'kov, and I. E. Dzyaloshinski, Methods of Quantum Field Theory in Statistical Physics (Dover, New York, 1975), p. 79.

${ }^{73}$ A. Fetter and J. D. Walecka, Quantum Theory of Many-Particle Systems (McGraw-Hill, New York, 1971), p. 401.

${ }^{74}$ S. V. Vonsovsky, Y. A. Izyumov, and E. Z. Kurmaev, Superconductivity of Transition Metals (Springer-Verlag, Berlin, 1982), Chap. 2.

${ }^{75} \mathrm{G}$. Grimvall, The Electron-Phonon Interaction in Metals (NorthHolland, Amsterdam, 1981), p. 4.

${ }^{76}$ P.A. Lee and T.V. Ramakrishnan, Rev. Mod. Phys. 57, 287 (1985).

${ }^{77}$ H. Gutfreund, M. Weger, and O. Entin-Wohlman, Phys. Rev. B 31, 606 (1985).

${ }^{78}$ Yong-Jihn Kim and K.J. Chang, Mod. Phys. Lett. B 12, 763 (1998).

${ }^{79}$ H. Lutz, H. Weismann, O.F. Kammerer, and M. Strongin, Phys. Rev. Lett. 36, 1576 (1976).

${ }^{80}$ H. Wiesmann, M. Gurvitch, H. Lutz, A. Ghosh, B. Schwarz, M. Strongin, P.B. Allen, and J.W. Halley, Phys. Rev. Lett. 38, 782 (1977).

${ }^{81}$ T.A. Miller, M. Kunchur, Y.Z. Zhang, P. Lindenfeld, and W.L. McLean, Phys. Rev. Lett. 61, 2717 (1988).

${ }^{82}$ A.M. Goldman and Y. Liu, Physica D 83, 613 (1995). 